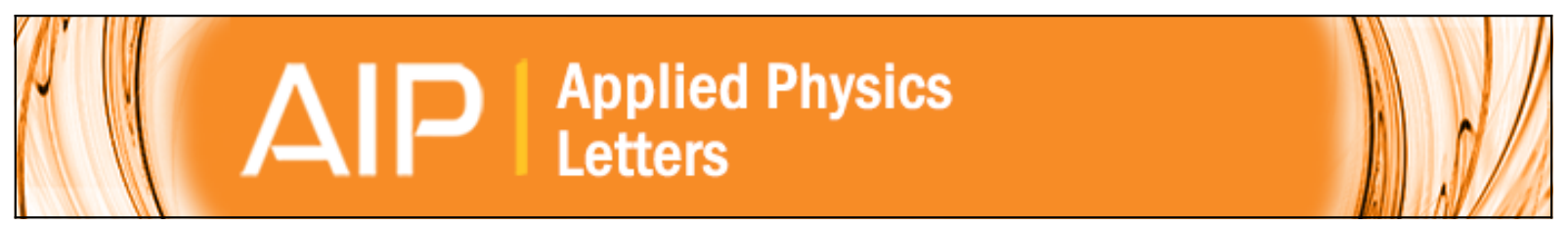

\title{
Ultrathin, epitaxial cerium dioxide on silicon
}

Jan Ingo Flege, Björn Kaemena, Jan Höcker, Florian Bertram, Joachim Wollschläger, Thomas Schmidt, and Jens Falta

Citation: Applied Physics Letters 104, 131604 (2014); doi: 10.1063/1.4870585

View online: http://dx.doi.org/10.1063/1.4870585

View Table of Contents: http://scitation.aip.org/content/aip/journal/apl/104/13?ver=pdfcov

Published by the AIP Publishing

\section{Articles you may be interested in}

Epitaxial, well-ordered ceria/lanthana high-k gate dielectrics on silicon

J. Vac. Sci. Technol. B 32, 03D124 (2014); 10.1116/1.4876122

Ge interface engineering using ultra-thin $\mathrm{La} 2 \mathrm{O} 3$ and $\mathrm{Y} 2 \mathrm{O} 3$ films: A study into the effect of deposition temperature J. Appl. Phys. 115, 114102 (2014); 10.1063/1.4868091

Epitaxial growth of $\mathrm{CeO} 2(111)$ film on $\mathrm{Ru}(0001)$ : Scanning tunneling microscopy (STM) and x-ray photoemission spectroscopy (XPS) study

J. Chem. Phys. 140, 044711 (2014); 10.1063/1.4849595

X-ray photoelectron diffraction study of dopant effects in La0.7X0.3MnO3 (X=La, Sr, Ca, Ce) thin films J. Appl. Phys. 113, 063511 (2013); 10.1063/1.4789988

Epitaxial growth and properties of Mo O x ( $2 \times 2.75)$ films

J. Appl. Phys. 97, 083539 (2005); 10.1063/1.1868852

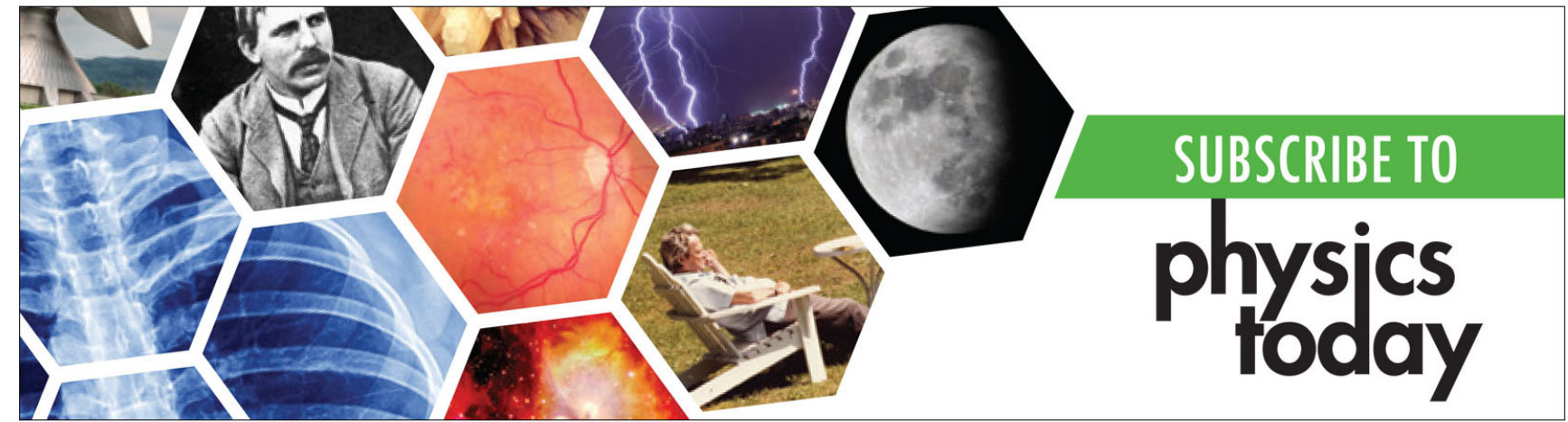




\title{
Ultrathin, epitaxial cerium dioxide on silicon
}

\author{
Jan Ingo Flege, ${ }^{1, a)}$ Björn Kaemena, ${ }^{1}$ Jan Höcker, ${ }^{1}$ Florian Bertram, ${ }^{2}$ Joachim Wollschläger, ${ }^{3}$ \\ Thomas Schmidt, ${ }^{1}$ and Jens Falta ${ }^{1}$ \\ ${ }_{1}^{1}$ Institute of Solid State Physics, University of Bremen, Otto-Hahn-Allee 1, 28359 Bremen, Germany \\ ${ }^{2}$ Photon Science, Deutsches Elektronensynchrotron (DESY), Notkestraße 85, 22607 Hamburg, Germany \\ ${ }^{3}$ Department of Physics, University of Osnabrück, Barbarastraße 7, 49069 Osnabrück, Germany
}

(Received 20 January 2014; accepted 25 March 2014; published online 2 April 2014)

It is shown that ultrathin, highly ordered, continuous films of cerium dioxide may be prepared on silicon following substrate prepassivation using an atomic layer of chlorine. The as-deposited, few-nanometer-thin $\mathrm{Ce}_{2} \mathrm{O}_{3}$ film may very effectively be converted at room temperature to almost fully oxidized $\mathrm{CeO}_{2}$ by simple exposure to air, as demonstrated by hard $\mathrm{X}$-ray photoemission spectroscopy and X-ray diffraction. This post-oxidation process essentially results in a negligible loss in film crystallinity and interface abruptness. (C) 2014 AIP Publishing LLC.

[http://dx.doi.org/10.1063/1.4870585]

Rare-earth oxides (REOs) show a wealth of intriguing physical and chemical properties, which arise from the unfilled $4 f$ electron shell of the metal cation and which are suitable for various kinds of technological applications. In addition to their use in research thrust areas like heterogeneous catalysis, renewable energy conversion, and storage, ${ }^{1}$ some of the REO have attracted a lot of interest in the field of microelectronics due to their high static dielectric constants $k$ and comparatively large band gaps, making them promising alternative materials for "high- $k$ " gate dielectrics replacing the traditional $\mathrm{SiO}_{2}{ }^{2-4}$ To this end, a particularly interesting candidate is $\mathrm{CeO}_{2}$, whose dielectric constant ${ }^{5,6}$ exceeds a value of $k>26$ and which exhibits a band $\operatorname{gap}^{7,8}$ of $\sim 6 \mathrm{eV}$. Furthermore, the almost perfect lattice match between silicon and $\mathrm{CeO}_{2}\left(\Delta a_{0} / a_{0}=0.36 \%\right)$ suggests the possibility of realizing an epitaxial, well-ordered, and sharp ceria-silicon interface. These gate oxide layers promise prolonged downscaling of field-effect transistors (FETs) by simultaneously increasing device performance and lowering power consumption. ${ }^{9}$ However, the interface between the silicon substrate and the high- $k$ gate oxide requires precise engineering since the interface trap densities and the carrier scattering need to be minimized to achieve reliable, high-performance devices. ${ }^{10}$ Yet, achieving a well-defined epitaxial interface has so far been considerably impeded by Ce-promoted silicon oxidation, resulting in amorphous silicon oxide and cerium silicate formation. ${ }^{11-15}$

To overcome the challenges posed by the high reactivity of the ceria-silicon interface, ${ }^{16}$ different approaches have been employed, ranging from influencing the growth kinetics by varying the REO growth rate and substrate temperature, the use of oxide buffer layers as, e.g., $\mathrm{CaF}_{2}{ }^{17}$ or $\mathrm{Pr}_{2} \mathrm{O}_{3}(0001),{ }^{18,19}$ to introducing surface active agents as, e.g., hydrogen. ${ }^{6,20,21}$ In this respect, passivation seems very promising since it may effectively facilitate the suppression of in-gap states associated with oxygen defects at the ceria-silicon interface. ${ }^{5}$ However, hydrogen limits the growth temperature to $450{ }^{\circ} \mathrm{C}$ due to its relatively low desorption temperature, ${ }^{22}$ motivating the search for other

a)Electronic mail: flege@ifp.uni-bremen.de adsorbates that would allow for higher growth temperatures because at given interface stability higher growth temperatures directly translate into an increased crystallinity of the deposited REO film.

Recently, we have shown that the use of chlorine, which is commonly used in semiconductor processing ${ }^{23}$ for substrate passivation enables the growth of well-ordered $\mathrm{Ce}_{2} \mathrm{O}_{3}(111)$ adlayers on $\mathrm{Si}(111)$ by reactive molecular beam epitaxy (MBE) in ultra-high vacuum (UHV), with $\mathrm{Cl}$ predominantly remaining at the oxide-silicon interface. ${ }^{24}$ Here, we apply the passivation-based approach and demonstrate the growth of few nm thick $\mathrm{Ce}_{2} \mathrm{O}_{3}(111)$ films and their conversion to $\mathrm{CeO}_{2}(111)$ while preserving an abrupt REO-silicon interface.

The experiments were performed both at the University of Bremen and at the Hamburg Synchrotron Radiation Laboratory (HASYLAB/DESY). After preparation of the samples under UHV conditions, X-ray diffraction (XRD) and grazing-incidence XRD (GIXRD) were conducted at the insertion device beamline BW2 using a primary photon energy of $10 \mathrm{keV}$ and a six-circle diffractometer in $z$-axis geometry. Atomic force microscopy (AFM) data were collected ex situ using a commercial microscope (NT-MDT) in tapping mode, also at HASYLAB. In addition, X-ray photoelectron spectroscopy (XPS) data were recorded in Bremen after exposure to air employing a dual X-ray anode combined with a hemispherical analyzer and a 7-channel detector (Omicron).

Sample preparation started from polished commercial $\mathrm{Si}(111)$ wafers, which were introduced into the UHV chamber and degassed at a temperature of $630^{\circ} \mathrm{C}$ for at least $12 \mathrm{~h}$. Flash-annealing to $1200^{\circ} \mathrm{C}$ removed the protective silicon oxide layer and established a sharp $(7 \times 7)$ reconstruction as verified by low-energy electron diffraction (LEED). Si(111) surfaces were passivated by exposure to molecular chlorine at $595^{\circ} \mathrm{C}$ using an electrochemical $\mathrm{AgCl}$ source as described earlier. ${ }^{25,26}$ Cerium oxide deposition was achieved by evaporating metallic $\mathrm{Ce}$ with an electron-beam evaporator in a preset oxygen partial pressure of $1-5 \times 10^{-7}$ millibars and at a substrate temperature of $500^{\circ} \mathrm{C}$. Typical growth rates were in the range of $2 \AA / \mathrm{min}$ as determined from complementary 

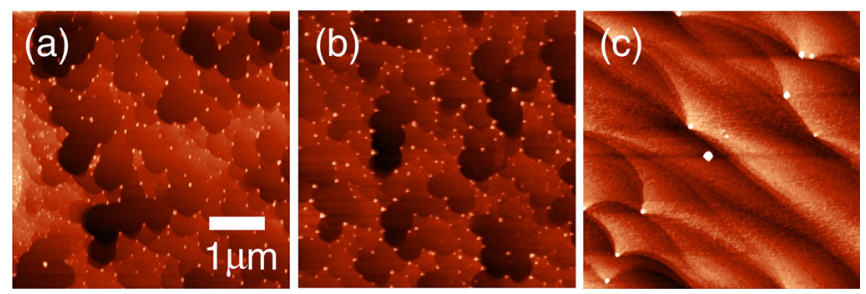

FIG. 1. AFM data acquired from cerium oxide films grown by reactive MBE on (a) bare $\mathrm{Si}(111)-(7 \times 7)$ and (b), (c) Cl-passivated $\mathrm{Si}(111)-(1 \times 1)$. The film thicknesses are (a), (b) $\sim 4 \mathrm{~nm}$ and (c) $18 \mathrm{~nm}$.

X-ray reflectometry (XRR) measurements. In reference experiments, cerium oxide films were directly deposited onto the $(7 \times 7)$ reconstructed $\mathrm{Si}(111)$ surface.

Typical AFM data recorded for cerium oxide films for various growth recipes are displayed in Figs. 1(a)-1(c). In the micrographs representing cerium oxide films (thickness $\sim 4 \mathrm{~nm}$ ) that have been grown on the bare (Fig. 1(a)) and the Cl-passivated (Fig. 1(b)) surface, smooth, wide terraces are observed. These terraces are separated by few-layer steps (height $\sim 1-2 \mathrm{~nm}$ ), which appear to be occasionally pinned by contaminants. Hence, we conclude that the film is essentially continuous and that its morphology remains unaffected by $\mathrm{Cl}$ preadsorption. Furthermore, the oxide terraces become even larger and smoother upon continued growth, albeit at the cost of more prominent step bunching, whereas the number of pinning centers is decreased considerably, as shown for an $18 \mathrm{~nm}$ thick film (Fig. 1(c)).

While there is no apparent difference in the surface morphology of the oxide films grown with and without substrate passivation, a significantly different behavior is noted regarding the oxidation state of the samples after exposure to air at room temperature, which is readily deduced from XPS data collected of the $\mathrm{Ce} 3 d$ and $\mathrm{O} 1 s$ core level regions (Fig. 2). Generally, the $\mathrm{Ce} 3 d$ electron configuration allows distinguishing between $\mathrm{Ce}$ atoms in different environments, i.e., between the $\mathrm{Ce}^{3+}\left(\mathrm{Ce}_{2} \mathrm{O}_{3}\right.$, Si-O-Ce silicate species) and $\mathrm{Ce}^{4+}\left(\mathrm{CeO}_{2}\right)$ cationic oxidation states. ${ }^{27,28}$ Since the $\mathrm{O} / \mathrm{s}$ core level is sensitive to changes in the coordination of the oxygen atoms, it additionally allows distinguishing between oxygen atoms in the different cerium oxides $\mathrm{CeO}_{2}$ and $\mathrm{Ce}_{2} \mathrm{O}_{3}{ }^{21}$ Here, we will use this property of the $\mathrm{O} / s$ level to determine the average oxidation state, i.e., the oxide stoichiometry, in the near-surface region. The uncertainties given below represent conservative estimates based on the overall robustness of the constrained least-square fitting procedures applied within independent $\mathrm{Ce} 3 d$ and $\mathrm{O} 1 s$ analyses, the latter accounting for the presence of contaminant species after air exposure, i.e., hydroxyls, carbonates, and water. ${ }^{29,30}$

Whereas in-situ XPS clearly reveals a sample stoichiometry of about $\mathrm{CeO}_{1.5}$ after growth at an oxygen partial pressure of up to $5 \times 10^{-7}$ millibars, both oxide films show substantial relative $\mathrm{Ce}^{4+}$ contributions after exposure to air, as consistently documented by the increased intensity of $\mathrm{Ce}^{4+}$-related components in the $\mathrm{Ce} 3 d^{28}$ and $\mathrm{O} 1 s^{21}$ spectra (Fig. 2). From the Ols data, average oxide stoichiometries of $\mathrm{CeO}_{1.88 \pm 0.05}$ in case of Cl-passivation and $\mathrm{CeO}_{1.64 \pm 0.05}$ for growth on bare $\mathrm{Si}$ are obtained for the near-surface region, clearly proving a much higher conversion rate to $\mathrm{Ce}^{4+}$ for the passivated substrates upon post-oxidation at room temperature.

We now turn to the characterization of the crystalline structure of the films and the effect of post-oxidation on their structural properties using XRD. Reciprocal space will be described using surface coordinates, i.e., in-plane lattice vectors in $[11 \overline{2}]$ and $[\overline{1} 2 \overline{1}]$ direction and out-of-plane in [111] direction with layer spacing periodicity, and the scattering vector $(H K L)$ will be referenced to the same coordinate system.

Fig. 3(a) shows XRD data of cerium oxide films that were grown at $500^{\circ} \mathrm{C}$ at an $\mathrm{O}_{2}$ partial pressure of $1 \times 10^{-7}$ millibars and sequentially capped by an amorphous layer of Si. Around the specular (111) bulk Bragg diffraction condition for bulk silicon, which, in surface coordinates, is located at (00 $L)_{\text {surf }}$ with $L=1$, Bragg peaks associated with the oxide films are found, demonstrating the crystallinity of the films in both cases. Furthermore, pronounced lattice fringes are clearly visible for oxide growth with and without Cl-passivation. These periodic oscillations are a clear sign that the films exhibit homogeneous film thicknesses of $5.9 \mathrm{~nm}$ (with Cl-passivation) and $5.1 \mathrm{~nm}$ (without passivation), respectively. From the position of the Bragg peaks associated with the oxide film, layer periodicities in [111] direction of $3.27 \AA$ and $3.28 \AA$ are deduced, which are
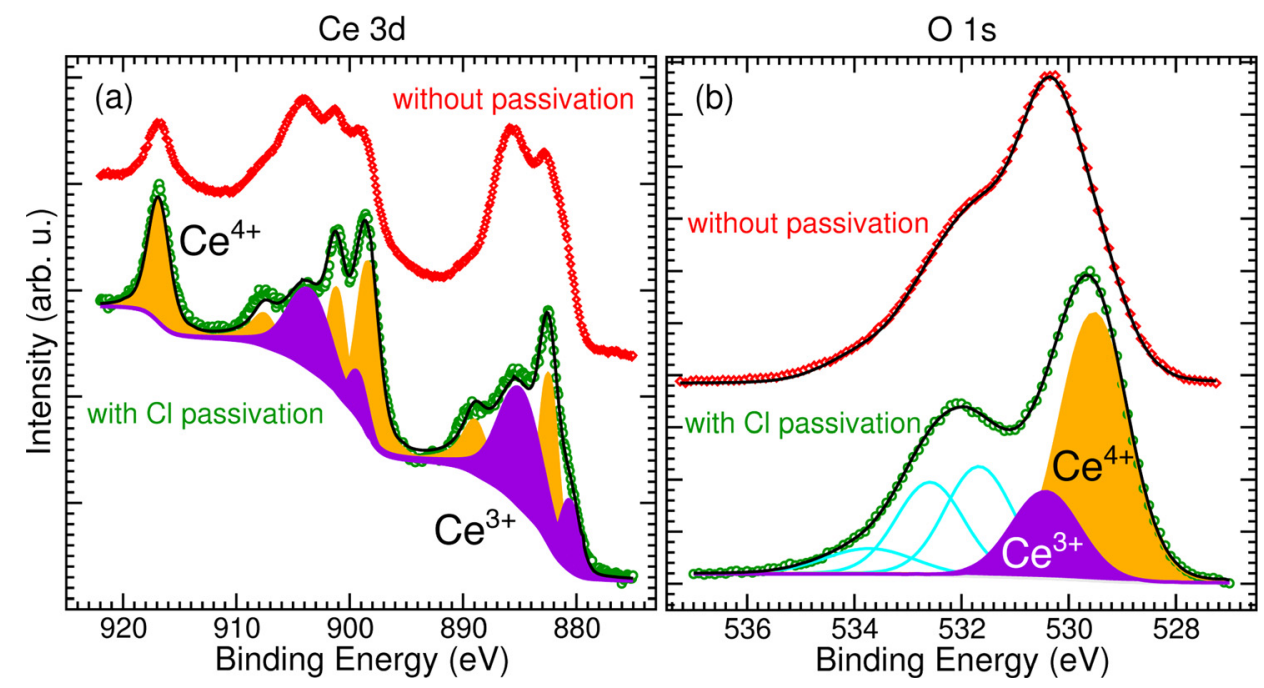

FIG. 2. Ex-situ Ce3d (a) and Ols (b) XPS data acquired after exposure to air from cerium oxide films grown by reactive MBE at identical conditions on bare and Cl-passivated $\mathrm{Si}(111)$. Constrained least-square fits (black) employing deconvolutions into various characteristic species are indicated as follows: (a) $\mathrm{Ce}^{3+}$ (violet), $\mathrm{Ce}^{4+}$ (orange); (b) $\mathrm{CeO}_{2}$ (orange), $\mathrm{Ce}_{2} \mathrm{O}_{3}$ (violet), and contaminants (cyan). 
cerium oxide films (as grown)

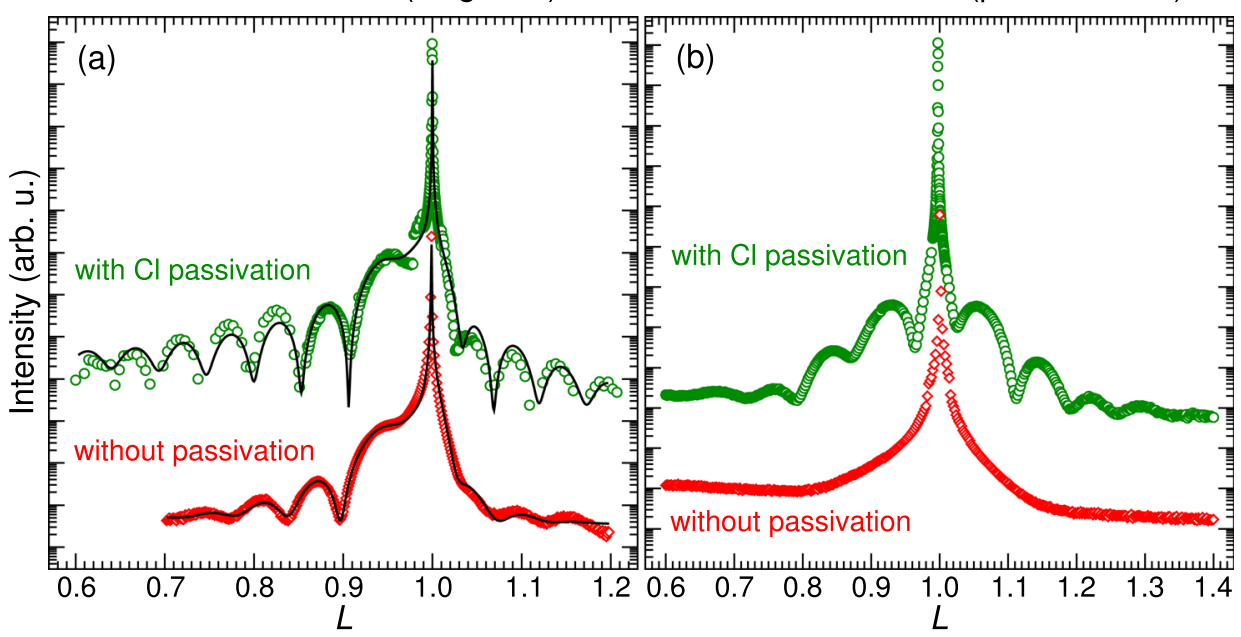

FIG. 3. XRD data (open symbols) and theoretical fit (solid lines) obtained for cerium oxide films on passivated and non-passivated substrates. (a) Samples capped with amorphous $\mathrm{Si}$, (b) after exposure to air (without capping). virtually identical within the error bar. These values are incompatible with the presence of a hexagonal $\mathrm{Ce}_{2} \mathrm{O}_{3}(0001)$ film, but they are very close to the calculated layer periodicity of $3.30 \AA$ based on elasticity theory using published elastic constants for $\mathrm{CeO}_{2}(111)^{31}$ and assuming a pseudomorphic, cubic $\mathrm{Ce}_{2} \mathrm{O}_{3}(111)$ oxide film, i.e., a film that has crystallized in the bixbyite structure and which is compressively strained to the substrate. Furthermore, we note that especially in the case of $\mathrm{Cl}$ passivation, the thickness oscillations decay very slowly with increasing distance from the Bragg peak, which bears clear testimony to a homogeneous film thickness combined with a rather low surface and interface roughness.

The conclusion on a sharper silicon/oxide interface for cerium oxide growth onto the Cl-terminated $\mathrm{Si}(111)$ surface from qualitative considerations is confirmed by quantitative analysis within the framework of the kinematical theory of $\mathrm{X}$-ray diffraction taking into account the potential existence of an amorphous interface layer between the substrate and the oxide grown on-top, resulting in an additional, interfacedependent phase shift of the reflected beam with respect to the incident beam. ${ }^{32}$ The respective fits to the experimental data [Fig. 3(a)] yield a decreased interface thickness of $0.4 \mathrm{~nm}$ for the Cl-passivated sample while for growth on the bare Si substrate it is $1.7 \mathrm{~nm}$ thick, again corroborated by independent XRR analysis.

Although substrate passivation yields superior interfacial properties, the preceding XRD analysis of the capped samples suggests that cubic $\mathrm{Ce}_{2} \mathrm{O}_{3}(111)$ films of reasonable crystalline quality may even be prepared on bare $\mathrm{Si}(111)$. However, a striking difference is observed when uncapped oxide films are exposed to ambient conditions, as demonstrated by the respective (00) crystal truncation rods (CTRs) (Fig. 3(b)). When grown on the bare substrate, the crystalline film thickness of the cerium oxide is severely diminished upon post-oxidation even at room temperature, as evidenced by a substantially broadened Bragg peak. Furthermore, the complete absence of thickness oscillations points to substantial roughening of the oxide-silicon interface since the surface roughness is not significantly influenced by Cl-passivation (cf. AFM data in Fig. 1). The broadened Bragg peak is centered around $L \approx 0.978$, demonstrating that the Ce oxidation state within the film is still relatively close to " $3+$." In case of substrate passivation, however, the Bragg peak has shifted to higher values closer to $L \approx 1.0$, which is expected for a Ce oxidation state approaching " $4+$ " within the oxide film. Also, the thickness fringes are preserved, clearly underlining that the high crystallinity and low roughness are maintained after exposure to air.

The quantitative XRD analysis of the (00)-CTR data of the post-oxidized film grown on the passivated substrate (Fig. 3(b)) reveals a film thickness of $3.5 \mathrm{~nm}$ and a vertical layer spacing of $3.16 \AA$ in the [111] direction, which is noticeably expanded as compared to the value of $3.12 \AA$ of bulk $\mathrm{CeO}_{2}$ (111) and considerably smaller than the respective layer spacing of $3.22 \AA$ found in bulk cubic $\mathrm{Ce}_{2} \mathrm{O}_{3}(111)$. Also, the abrupt nature of the oxide-silicon interface is shown to be preserved, as documented by its almost vanishing thickness of only $0.3 \pm 0.1 \mathrm{~nm}$, which is the same as previously found for capped samples.

Details about the stacking sequence of the film as well as the respective in-plane lattice constant can be determined from grazing-incidence XRD. The (01) CTR data (Fig. 4(a)) indicate that only so-called $B$-type stacking is observed, i.e., a stacking fault is introduced at the oxide-silicon interface, which has already been found for cerium oxide grown on

(a) (01) CTR

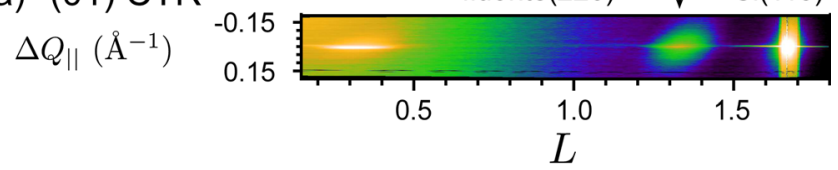

(b) $\left(\begin{array}{lll}0 & 1 & 1.33)\end{array}\right.$

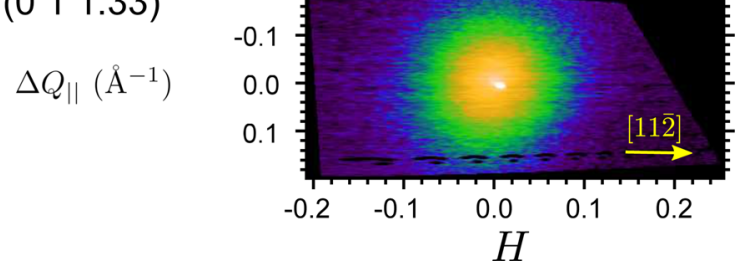

FIG. 4. GIXRD data obtained for cerium oxide growth on Cl-passivated $\mathrm{Si}(111)$ at $5 \times 10^{-7}$ millibars $\mathrm{O}_{2}$ backfilling and a substrate temperature of $500{ }^{\circ} \mathrm{C}$ and subsequent exposure to air: (a) $\mathrm{Si}(01 L)$ CTR data and (b) in-plane reciprocal space map recorded by cutting through the $(01 L)$ rod at $L=1.33$. 
(a)

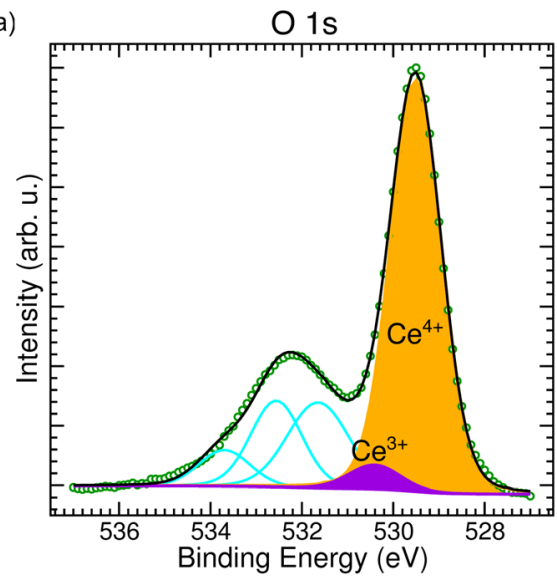

(b) (01) CTR

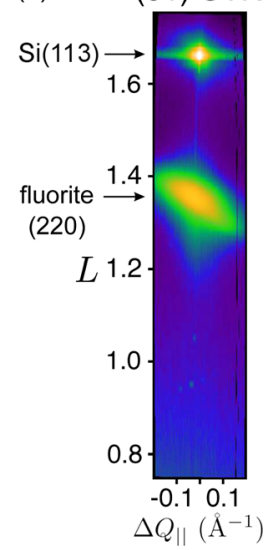

FIG. 5. XPS and GIXRD data recorded for an $18 \mathrm{~nm}$ thick cerium oxide film grown at $5 \times 10^{-7}$ millibars and a substrate temperature of $500^{\circ} \mathrm{C}$ and subsequently exposed to air. (a) $\mathrm{O} 1 s$ data (circles) and fit using the deconvolution introduced before, including $\mathrm{Ce}_{2} \mathrm{O}_{3}$ (violet) and $\mathrm{CeO}_{2}$ (orange) components. (b) (01)-CTR data demonstrating the presence of tilt mosaicity in the fully oxidized ceria film.

hydrogen-passivated silicon. ${ }^{33}$ Furthermore, the in-plane reciprocal space map recorded by cutting through the $(01)$ CTR at $L=1.33$ (Fig. 4(b)) demonstrates that the ceria film is fully strained to the substrate. Moreover, the reflection appears to be perfectly circular, ruling out the presence of twist mosaicity.

For an $18 \mathrm{~nm}$ thick film grown at the same conditions and post-oxidized in air, both XPS and GIXRD corroborate the presence of fully oxidized ceria. From quantitative $\mathrm{O} 1 \mathrm{~s}$ peak fitting (Fig. 5(a)) a near-surface stoichiometry of $\mathrm{CeO}_{1.98}$ is estimated, while XPS employing hard X rays indicates the presence of $\mathrm{CeO}_{2}$ in the deeper layers. The analysis of the (01)-CTR data (Fig. 5(b)) yields a (111) layer spacing of $3.09 \pm 0.01 \AA$, in very good agreement with the predicted layer spacing of $3.11 \AA$ based on simulations for a pseudomorphic $\mathrm{CeO}_{2}$ film using elasticity theory. Furthermore, the elliptical smearing of the $\left(01 \frac{4}{3}\right)$ oxide reflection clearly points to the formation of oxide domains that are inclined by $(1.3 \pm 0.2)^{\circ}$ with respect to the [111] direction. This tilt mosaicity, however, is absent for ultrathin films (Fig. 4(a)).

Concluding, it was demonstrated that well-ordered, epitaxial cerium oxide films may be formed from well-defined $\mathrm{Ce}_{2} \mathrm{O}_{3}$ films on $\mathrm{Cl}$-passivated $\mathrm{Si}(111)$ grown by $\mathrm{MBE}$. Already at room temperature, these few-nanometer thin films can be postoxidized close to the $\mathrm{CeO}_{2}$ stoichiometry by simple exposure to air, showcasing the high oxygen mobility and storage capacity facilitated by the structural quality of the oxide. During the structural transformation the abrupt nature of the ceria-silicon interface is preserved, also suggesting use in future FET based, chemical sensing technologies.

Portions of this research were carried out at the light source DORIS III at DESY. DESY is a member of the Helmholtz Association (HGF). We would like to thank Dmitri Novikov for assistance in using beamline BW2,

Sebastian Gevers for assistance in quantitative XRD analysis and the COST Action CM1104 for partial support.

${ }^{1}$ Catalysis by Ceria Related Materials, edited by A. Trovarelli (Imperial College Press, 2001).

${ }^{2}$ Rare Earth Oxide Thin Films, Topics in Applied Physics, edited by M. Fanciulli and G. Scarel (Springer, Berlin, Heidelberg, 2007).

${ }^{3}$ D. G. Schlom, S. Guha, and S. Datta, Mater. Res. Bull. 33, 1017 (2008).

${ }^{4}$ H. J. Osten, A. Laha, M. Czernohorsky, E. Bugiel, R. Dargis, and A. Fissel, Phys. Status Solidi C 205, 695 (2008).

${ }^{5}$ Y. Nishikawa, T. Yamaguchi, M. Yoshiki, H. Satake, and N. Fukushima, Appl. Phys. Lett. 81, 4386 (2002).

${ }^{6}$ Y. Nishikawa, N. Fukushima, N. Yasuda, K. Nakayama, and S. Ikegawa, Jpn. J. Appl. Phys., Part 1 41, 2480 (2002).

${ }^{7}$ D. D. Koelling, A. M. Boring, and J. H. Wood, Solid State Commun. 47, 227 (1983).

${ }^{8}$ E. Wuilloud, B. Delley, W.-D. Schneider, and Y. Baer, Phys. Rev. Lett. 53, 202 (1984).

${ }^{9}$ High Dielectric Constant Materials: VLSI MOSFET Applications, Springer Series in Advanced Microelectronics, edited by H. Huff and D. Gilmer (Springer, Berlin, Heidelberg, 2004).

${ }^{10}$ G. D. Wilk, R. M. Wallace, and J. M. Anthony, J. Appl. Phys. 89, 5243 (2001).

${ }^{11}$ F. U. Hillebrecht, M. Ronay, D. Rieger, and F. J. Himpsel, Phys. Rev. B 34, 5377 (1986).

${ }^{12}$ W. A. Henle, M. G. Ramsey, F. P. Netzer, R. Cimino, W. Braun, and S. Witzel, Phys. Rev. B 42, 11073 (1990).

${ }^{13}$ T. Chikyow, S. M. Bedair, L. Tye, and N. A. El-Masry, Appl. Phys. Lett. 65, 1030 (1994).

${ }^{14}$ E. J. Preisler, O. J. Marsh, R. A. Beach, and T. C. McGill, J. Vac. Sci. Technol., B 19, 1611 (2001).

${ }^{15}$ V. Narayanan, S. Guha, M. Copel, N. A. Bojarczuk, P. L. Flaitz, and M. Gribelyuk, Appl. Phys. Lett. 81, 4183 (2002).

${ }^{16}$ S. Stemmer, J. Vac. Sci. Technol., B 22, 791 (2004).

${ }^{17}$ J. Zarraga-Colina, R. M. Nix, and H. Weiss, Surf. Sci. 563, L251 (2004).

${ }^{18}$ M. H. Zoellner, J. Dabrowski, P. Zaumseil, A. Giussani, M. A. Schuber, G. Lupina, H. Wilkens, J. Wollschläger, M. Reichling, M. Bäumer, and T. Schroeder, Phys. Rev. B 85, 035302 (2012).

${ }^{19}$ H. Wilkens, O. Schuckmann, R. Oelke, S. Gevers, A. Schaefer, M. Bäumer, M. H. Zoellner, T. Schroeder, and J. Wollschläger, Appl. Phys. Lett. 102, 111602 (2013).

${ }^{20}$ M. Yoshimoto, K. Shimozono, T. Maeda, T. Ohnishi, M. Kumagai, T. Chikyow, O. Ishiyama, M. Shinohara, and H. Koinuma, Jpn. J. Appl. Phys., Part 2 34, L688 (1995).

${ }^{21}$ B. Hirschauer, M. Göthelid, E. Janin, H. Lu, and U. O. Karlsson, Appl. Surf. Sci. 148, 164 (1999).

${ }^{22}$ P. Gupta, V. L. Colvin, and S. M. George, Phys. Rev. B 37, 8234 (1988).

${ }^{23}$ M. L. Yu and L. A. DeLouise, Surf. Sci. Rep. 19, 285 (1994).

${ }^{24}$ J. I. Flege, B. Kaemena, S. Gevers, F. Bertram, T. Wilkens, D. Bruns, J. Bätjer, T. Schmidt, J. Wollschläger, and J. Falta, Phys. Rev. B 84, 235418 (2011).

${ }^{25}$ J. I. Flege, T. Schmidt, J. Falta, and G. Materlik, Surf. Sci. 507-510, 381 (2002).

${ }^{26}$ J. I. Flege, T. Schmidt, J. Bätjer, M. Çakmak, J. Falta, and G. Materlik, New J. Phys. 7, 208 (2005).

${ }^{27}$ M. Romeo, K. Bak, J. E. Fallah, F. L. Normand, and L. Hilaire, Surf. Interface Anal. 20, 508 (1993).

${ }^{28}$ D. R. Mullins, S. H. Overbury, and D. R. Huntley, Surf. Sci. 409, 307 (1998).

${ }^{29}$ L. Kundakovic, D. R. Mullins, and S. H. Overbury, Surf. Sci. 457, 51 (2000).

${ }^{30}$ S. D. Senanayake and D. R. Mullins, J. Phys. Chem. C 112, 9744 (2008).

${ }^{31}$ A. Nakajima, A. Yoshihara, and M. Ishigame, Phys. Rev. B 50, 13297 (1994).

${ }^{32}$ T. Weisemoeller, F. Bertram, S. Gevers, C. Deiter, A. Greuling, and J. Wollschläger, Phys. Rev. B 79, 245422 (2009).

${ }^{33}$ M. Furusawa, J. Tashiro, A. Sasaki, K. Nakajima, M. Takakura, T. Chikyow, P. Ahmet, and M. Yoshimoto, Appl. Phys. Lett. 78, 1838 (2001). 